\title{
THE PECULIAR STARS AND SPECTRAL CLASSIFICATION
}

\author{
E. K. KHARADZE and R. A. BARTAYA \\ Abastumani Astrophysical Observatory, U.S.S.R.
}

(Read by B. E. Westerlund)

\begin{abstract}
The spectral classification experiments with the 70-cm meniscus telescope and $8^{\circ}$ objective prism of Abastumani Observatory (dispersion $166 \AA \mathrm{mm}^{-1}$ ) have shown that if we apply the Kodak IIa-O plates at the $0.4 \mathrm{~mm}$ widening of spectra and the 20 minutes exposure time, we can successfully classify with relatively high accuracy in two dimensional MK system the stars down to the 11th magnitude, with simultaneous discrimination of peculiars.

We have undertaken such determinations first of all in Kapteyn Selected Areas aiming to fill the gaps in existing data for these standard areas.

Finally we could mention the high quality of the meniscus telescope optics together with good seeing in Abastumani as favourable factors making the Abastumani spectra of 10-11th magn. stars comparable with the slit spectra of the same dispersion.

The Abastumani spectra allow to apply the Morgan and Kitt-Peak criteria of MK classification only with a slight modification.

We have two-dimensionally classified in MK system about ten thousand stars down to the 11th magnitude in KA Nos. 2-43 $\left(4.5 \times 4^{\circ} 5\right.$ each). Among them 137 peculiar stars have been revealed. They are mainly Ap, Am and Ball (7 stars) and the stars with composed spectra (5). 97 stars have been first identified as peculiars.
\end{abstract}

It may be stated that there is a general growth of interest now in the peculiar stars, i.e. the stars having some sorts of anomalies in their spectra.

Not only the probable connection of peculiarities with the physical state and evolutionary processes in stars raises the interest. Simply it seems impossible now to make sufficiently accurate spectral classification if the stars belonging to a definite spectral group are not preliminary discriminated.

As a matter of fact the accuracy of the two-dimensional classification is the higher the more uniform the group of classified stars is. But if this is not the case we have to deal with the multi-dimensional classification which is a more difficult task indeed.

Principally we may involve as many parameters as we find it necessary to have manifested all the peculiarities present in the spectra of stars. But the quantitative methods for such aim have not been so widely applied. Till now the aim of these methods consisted in two or three-dimensional classification applicable to the majority of stars.

If we remember also the difficulties attending the realization even of the three-dimensional classification we easily imagine the importance and the relative privilege of the qualitative classification, allowing the discrimination of the peculiars, i.e. the grouping the stars into uniform groups.

Only after such groups of stars have been composed they may be classified quantitatively and the rate of peculiarity may be determined together with the two common parameters.

Peculiarities are represented by very slight effects in spectra. Only the recent development of observational technique made it possible to study the peculiar stars on a large scale. The objective prism spectra serve well for the discoveries of peculiar stars. But the dispersion and the quality of such spectra are restricted. This requires that we 
consider and attack the problem individually in each case of the given technique and the given dispersion.

The spectral classification experiments with the 70-cm meniscus telescope and the $8^{\circ}$ objective prism of Abastumani Observatory (dispersion $166 \AA \mathrm{mm}^{-1}$; the edge of the spectrum on the short wave side at about $3500 \AA$ ) have shown that if we use the Kodak IIa-O plates, the $0.4 \mathrm{~mm}$ widening of spectra and the 20 -min exposure time, we can successfully classify with relatively high accuracy in two-dimensional MK system the stars down to the 11 th mag., with simultaneous discrimination of peculiars. This procedure is rather effective for peculiars of the types Ap, Am and BaII and for stars having composed spectra.

It scarcely requires to stress the importance of such work from the point of view of stellar astronomy problems.

We have undertaken such determinations first of all in Kapteyn Selected Areas aiming to fill the gaps in existing data for these standard areas. No doubt we remember well the widely known work by Fehrenbach, but the limiting star in his work is much brighter (about $9.5 \mathrm{mg}$ ). The definite advantage of Fehrenbach's determinations is the higher dispersion (80-100 $\AA$ ). Their uniformity should also be recognized. But Fehrenbach had been doing his work when the peculiars had not been attracting much attention. This fact inevitably introduced some errors of classification. We emphasize the necessity of preliminary discrimination of peculiar stars if we wish to secure high accuracy of the MK classification.

We would like to stress the full comparability of our results with those by Fehrenbach in spite of lower dispersion of our spectra. This may be ascribed to two facts.

Firstly we have used a shorter exposure time: 20 min vs Fehrenbach's $2 \mathrm{~h}$.

Secondly, the length of our spectra reaching $3500 \AA$ exceeds on the short-wave side that of Fehrenbach's $(3900 \AA)$. This difference arguing in our favour is of essential importance for early type stars and such as the peculiar stars Ap and Am.

Finally we mention the high quality of the meniscus telescope optics together with good seeing in Abastumani as favourable factors making the Abastumani slitless spectra of 10-11th mg stars comparable with the slit spectra of the same dispersion (West, 1970).

In the specified magnitude interval the Abastumani spectra allow to apply the Morgan and Kitt-Peak criteria of MK classification only with a slight modification.

As for the Kitt-Peak spectra, their dispersion $\left(128 \AA \mathrm{mm}^{-1}\right)$ does not differ from that of ours. Moreover: starting from $\mathrm{H} \delta$ down to the short-wave region, where the majority of criteria for the early types are present, the Abastumani spectral dispersion is even a little higher.

We have two-dimensionally classified in MK system about ten thousand stars down to the 11 th mag. in KA Nos. 2-43 (4.5 $\times 4.5$ each). Among them 137 peculiar stars have been revealed. They are mainly Ap, Am and BaII ( 7 stars) and the stars with composed spectra (5). Ninetyseven stars have been first identified as peculiars (Astron. Circ. Acad.Sci.U.S.S.R. No. 583, 1970). Let us now describe in short the criteria we have used.

Ap stars. These are stars from B5 to F0, but for the most part B9-A2, and of 
IV, V class luminosity. Si II $4128-30$ is always present. But sometimes Sr II 4077, Mn I 4030-33, Cr II 4171, Eu II 4205 (all or some of them) are visible too. As it is known, the 4128-30 $\AA$ region contains, besides Si II, the Eu II, Cr II, Fe I lines. It is difficult to state which of them prevail in any particular case. However, the integral effect is distinct enough to reveal the peculiarity, one never misses it.

In low dispersion spectra we do not see always all individual lines of the mentioned elements. Therefore we restrict ourselves to identifying the peculiars Ap, listing only the lines which are more or less distinctly visible without naming their elements.

One must remember that such a strong characteristics of Ap stars as the 4128-30 $\AA$ is also characteristic for supergiants. Therefore if one leaves out of account the hydrogen lines intensity and other characteristic lines of supergiants (especially distinguishable Ti II 3760, 3815), one might erroneously classify a peculiar as a supergiant.

In case of objective prism spectra of the so-called hot Ap stars we encounter some difficulties. As is known they are the early stars up to B5 in whose spectra the He lines are weakened. For this special reason one classifies them erroneously as B8, B9 stars and they are missed as peculiars. Therefore when one has to deal with stellar associations where the probability of B0-B5 stars presence is high by itself, one must be very careful classifying the stars. Generally speaking the suggestion is needed of some quantitative or at least qualitative characteristics to avoid such mistakes.

Am stars. Early type stars A0-A5 if determined according to $\mathrm{Ca}$ II $\mathrm{K}$; hydrogen lines are weakened if compared with common IV, V class stars, while the metal lines are strengthened.

In case of A5 and later subclasses it becomes difficult to discriminate peculiars from supergiants. This is evident from the Kitt-Peak spectra reproductions. As for the stars earlier than A5 along with other properties Sr II 4077 line favours the matter very much, which is quite an essential and strong feature for Am stars, while in our spectra this line is absent even in supergiants up to A5.

It must be mentioned, that both Ap and Am stars are divided into subtypes according to the ratio $\mathrm{K} / \mathrm{H}$. It is clear that to take $\mathrm{Ca}$ II $\mathrm{K}$ line for the Ap stars is not justified. But on the other hand this ratio somehow points to what this $\mathrm{Ca}$ II $\mathrm{K}$ line was like at the moment of our observation.

Ba II stars. G8-K0 Giants. Sr II 4077 and 4216 lines attract one's attention with their intensity. 4077 ( $\mathrm{Sr} \mathrm{II}) \gg 4063$ ( Fe I) and 4216 ( $\mathrm{Sr} \mathrm{II}) \geqslant 4226$ (Ca I). G band (CH) is strengthened as a rule. The $\mathrm{Ba}$ II 4554 line itself mostly characteristic to these stars is less distinct in our spectra on account of low dispersion.

It must be borne in mind that $4077 \AA$ and $4216 \AA$ are also strong in supergiants together with other characteristics, but they never attain the strength as they do in Ba II stars. Particularly, 4077 surpasses 4063 in intensity slightly and 4216 approaches but never equals 4226 .

It is noteworthy that we stress the resemblance of the properties of the Ap, Am, BaII stars with those of the supergiants, because it is this resemblance and its ignorance that have conditioned those inherent errors, which as mentioned above, are encountered in Fehrenbach Catalogue. 
We shall not concern ourselves with the stars of composed spectra for they are in general easily distinguished from 'normal' ones.

We should note, that all the peculiar stars from the Catalogues of Osawa (1965), Bertaud $(1959,1960)$ and from other wellknown sources, covered with our areas have shown themselves as peculiar ones on our negatives too. A few stars are exceptions, which happened to be overexposed owing to their considerable brightness, or as we believe, they were erroneously assigned to peculiar stars by other investigators for they might have been partially covered by adjacent spectra to judge by our plates of relatively large scale.

The results obtained show the efficiency of the work of this sort carried on with our technique. In addition to this we intend to study the areas under consideration from the point of view of searching for $\mathrm{H} \alpha$ emission stars and the red stars. This might give us a possibility to study the area more thoroughly. In particular the above-mentioned Kapteyn Areas Nos. 2-43 have already been covered with panchromatic Agfa Zp-3 plates (with the limiting magnitude about 13-14). The material is being treated.

An attempt was made to study the problem of galactic distribution of Ap, Am stars on the basis of our data. Though we have only about 120 stars, the distribution of the Kapteyn Areas themselves and the uniformity of data makes it possible to get some hints about the distribution of peculiars in the Galaxy.

Some of the Kapteyn Areas (Nos. 8, 9, 18, 19,40) coincide with associations and open clusters.

Ap stars clearly indicate the concentration towards the galactic plane $\left(-20^{\circ} \geqslant b \leqslant\right.$ $+20^{\circ}$ interval). But it cannot be said that they are much more grouped in associations. This may be attributed both to the scarcity of data and to the fact that in this respect these areas are not different from others. At any rate the solving of this problem requires much more plentyful material and the discrimination of hot Ap stars, which are more expected just in these areas.

As for Am stars, they show an equal distribution in percentage over the sky.

From the standpoint of investigation of the problems of Ap, Am stars we intend further to extend this type of work just in association areas.

At the same time, in order to be sure that the members of associations are completely involved we contemplate to investigate these areas additionally with $4^{\circ}$ prism (dispersion $660 \AA \mathrm{mm}^{-1}$ ). This will give us a possibility to separate the stars of the so-called $O B$ group fainter than 11 th mag.

As to the areas of open clusters and the problems of Ap, Am stars distribution in these open clusters Dr E. Zhelvanova (Potsdam, G.D.R.) is conducting this work using the Abastumani spectra.

\section{References}

Bertaud, C.: 1959, J. Obs. 42, 45.

Bertaud, C.: 1960, J. Obs. 43, 129.

Osawa, K.: 1965, Ann. Tokyo Astron. Obs., Ser. 2, 9, 123.

West, R.: 1970, Abastumani Bull. No. 39. 